\title{
Multi-Rank Adaptive Beamforming
}

\author{
Louis L. Scharf ${ }^{1,2}$, Ali Pezeshki ${ }^{1}$, and Magnus Lundberg ${ }^{3}$ \\ ${ }^{1}$ Department of Electrical and Computer Engineering \\ ${ }^{2}$ Department of Statistics \\ Colorado State University, Fort Collins, Co 80523 \\ ${ }^{3}$ Department of Computer Science and Electrical Engineering \\ Luleä University of Technology, Luleä, Sweden \\ \{scharf,ali\}@engr.colostate.edu,mlg@sm.luth.se
}

\begin{abstract}
This paper presents a multi-rank generalization of the Capon beamformer to accommodate model mismatch in situations where the unknown signal of interest lies in a multidimensional subspace. By expanding the beamforming subspace robustness (or diversity) is achieved at the expense of resolution. The generalization involves solving a quadratically-constrained quadratic minimization problem, and designing a constraint matrix. Three strategies for designing this constraint matrix are discussed. Simulation examples are presented to demonstrate the performance of the multi-rank Capon beamformer.
\end{abstract}

\section{INTRODUCTION}

Capon beamforming is widely used in radar, sonar, and digital communications. The standard Capon beamformer relies on the predictable and planar structure of the propagating wavefront. In many applications, however, the structure of the propagating wavefront is not planar. This may be due to calibration errors, uncertainties about the direction of arrival, local scattering, multi-path, etc. In such scenarios, mismatches between the presumed and actual signal models are known to degrade the performance of the Capon beamformer, unless proper measures are taken [1],[2].

To date, several approaches have been reported to robustify the Capon beamformer, among which are the robust adaptive beamformers of [3] and [4], and the robust Capon beamformers of [5] and [6]. These approaches are more or less similar, in the sense that the resultant beamformer is the principal eigenvector of a diagonally loaded version of $\mathbf{R}^{-1} \mathbf{R}_{s s}$, and is of rank-1. Here $\mathbf{R}$ and $\mathbf{R}_{s s}$ are data and signal covariance matrices, respectively.

In this paper, we develop a multi-rank extension of the Capon beamformer. The idea is that a beamforming method that maps the power in a multi-dimensional subspace is more likely to be robust to model mismatches than a method that maps the power in a one-dimensional subspace. Roughly speaking, a multi-dimensional subspace beamformer may be viewed as a matrix consisting of a collection of rank-1 beamformers that are matched to perfect planewaves at nearby angles. Therefore, we may think of the power out of a multirank beamformer as the average power of multiple rank-1

This work was supported by The Office of Naval Research under contract N00014-04-1-0084 and by The DARPA through The Air Force Office of Scientific Research grant FA9550-04-1-0371. beamformers over a range of angles. This seems like a diversity combining idea. However, averaging of powers results in loss of resolution. Thus, the downside of expanding the beamforming subspace is that robustness is achieved at the expense of resolution. Unlike the approaches of [3]-[6] where the Capon spectrum is determined by a single eigenvalue of $\mathbf{R}^{-1} \mathbf{R}_{s s}$, in our approach multiple eigenvalues of $\mathbf{R}^{-1} \mathbf{R}_{s s}$ determine the spectrum, each of which is weighted to meet a constraint.

The idea of forming multi-rank spectral estimators and beamformers is not new. In fact, the multiple window approach of Thomson [7],[8] may, in some sense, be viewed as a multirank extension of the Bartlett method. A multi-rank generalization of the Capon beamformer has been reported in [9] and [10], which involves a linearly-constrained quadratic minimization problem. Our multi-rank generalization of the Capon beamformer (also see [11]), however, involves a quadratic minimization under a quadratic power constraint. We have recently discovered a connection between these linearly- and quadratically-constrained minimization problems [12]. It turns out that the quadratically-constrained problem, which is nonconvex, can be solved by solving a set of linearly-constrained convex problems and then determining the best linear solution over the set of all linear solutions, which is convex with respect to orthogonal transformations. In addition, the solutions to both linearly- and quadratically-constrained problems may be cast in the form of oblique projections, for which there are illuminating circuit diagrams called generalized sidelobe cancellers [12].

In this paper, we limit our scope to developing a multirank extension of the Capon beamformer under a set of quadratic constraints, and evaluating its performance. The connection between our multi-rank Capon beamformer and the one reported in [9] and [10] is established in [12].

\section{PROBlem SETUP}

Consider the observation model

$$
\mathbf{x}=\mathbf{s}+\mathbf{n}
$$

where $\mathbf{s} \in \mathbb{C}^{n}$ is the source vector or signal of interest, with covariance $\mathbf{R}_{s s}=E\left[\mathbf{s s}^{H}\right], \mathbf{x} \in \mathbb{C}^{n}$ is the data vector, recorded 
by $n$ sensors, with covariance $\mathbf{R}=E\left[\mathbf{x x}^{H}\right]$, and $\mathbf{n} \in \mathbb{C}^{n}$ is a proper noise vector, with covariance $\mathbf{R}_{n n}=E\left[\mathbf{n n}^{H}\right]$.

In a perfect planewave case, the source vector $\mathbf{S}$ and its covariance matrix $\mathbf{R}_{s s}$ are modelled as

$$
\begin{gathered}
\mathbf{s}=\boldsymbol{\psi}(\theta) s=\mathbf{D}(\theta) \mathbf{1} s \\
\mathbf{R}_{s s}=\mathbf{R}_{s s}(\theta)=\boldsymbol{\psi}(\theta) \sigma_{s}^{2} \boldsymbol{\psi}(\theta)^{H}=\mathbf{D}(\theta) \mathbf{1} \sigma_{s}^{2} \mathbf{1}^{H} \mathbf{D}(\theta)^{H}
\end{gathered}
$$

where $s$ is a zero-mean complex normal variable with variance $\sigma_{s}^{2}=E\left[s^{2}\right], \quad \mathbf{1}^{H}=[1,1, \ldots, 1] / \sqrt{n}$ is the normalized bearing vector at broadside, and $\mathbf{D}(\theta)=$ $\operatorname{diag}\left(1, e^{j \pi \sin \theta}, \ldots, e^{j(n-1) \pi \sin \theta}\right)$ is the diagonal matrix that steers the wavefront to angle $\theta$, and $\psi(\theta)=\mathbf{D}(\theta) \mathbf{1}$.

In general, however, the wavefront $\mathbf{s}$ can be non-planar (wrinkled) and may be modelled as

$$
\mathbf{s}=\mathbf{D}(\theta) \int_{-\pi \beta}^{\pi \beta} \boldsymbol{\psi}\left(\theta^{\prime}\right) \mu\left(\theta^{\prime}, k\right) \frac{d \theta^{\prime}}{\sqrt{2 \pi \beta}}
$$

with covariance matrix

$\mathbf{R}_{s s}(\theta)=E\left[\mathbf{s s}^{H}\right]=\mathbf{D}(\theta)\left[\int_{-\pi \beta}^{\pi \beta} \boldsymbol{\psi}\left(\theta^{\prime}\right) \boldsymbol{\psi}\left(\theta^{\prime}\right)^{H} S\left(\theta^{\prime}\right) \frac{d \theta^{\prime}}{2 \pi \beta}\right] \mathbf{D}(\theta)^{H}$

where the $\mu\left(\theta^{\prime}, k\right)$ are uncorrelated random variables with powers $E\left[\mu\left(\theta^{\prime}, k\right)^{2}\right]=S\left(\theta^{\prime}\right)$ over the $(-\beta \pi, \beta \pi]$ angular bandwidth. In other words, the wavefront is produced in accordance to a power spectrum over a spread of angles. The signal covariance matrix in (5) may be shown to be of approximate rank $p=[\beta n]+1$, where $[\beta n]$ is the integer part of $\beta n$.

Assuming that $S$ is constant over the $(-\beta \pi, \beta \pi]$ angular bandwidth, the expressions for $\mathbf{s}$ and $\mathbf{R}_{s s}(\theta)$ may be approximated by

$$
\begin{gathered}
\mathbf{s}(\theta)=\mathbf{D}(\theta) \Phi \mathbf{v} \\
\mathbf{R}_{s s}(\theta)=\mathbf{D}(\theta) \boldsymbol{\Phi}(\theta) \mathbf{R}_{v v} \boldsymbol{\Phi}(\theta){ }^{H} \mathbf{D}(\theta)^{H}
\end{gathered}
$$

where $\boldsymbol{\Phi}=\left[\phi_{1}, \ldots, \phi_{p}\right] \in \mathbb{C}^{n \times p}$ is the matrix containing the first $p$ Slepian basis vectors, and $\mathbf{v}$ is a vector of random complex coefficients with covariance $\mathbf{R}_{v v}=E\left[\mathbf{v} \mathbf{v}^{H}\right]$. The complex vector $\boldsymbol{\Phi} \mathbf{v}$ is a non-planar (wrinkled) wavefront at broadside, which is steered to angle $\theta$ using $\mathbf{D}(\theta)$. In much of what follows we use the notations $\mathbf{R}_{s s}$ and $\boldsymbol{\psi}$, with the understanding that these are $\mathbf{R}_{s s}(\theta)$ and $\boldsymbol{\psi}(\theta)$.

To fully exploit the multi-dimensional signal subspace, it seems natural to map the power in a multi-dimensional subspace, using a multi-rank beamformer, rather than to map it in a one-dimensional subspace, using a rank-1 beamformer. The power out of a multi-rank beamformer, say $\mathbf{W}(\theta)=$ $\left[\mathbf{w}_{1}, \ldots, \mathbf{w}_{r}\right](\theta) \in \mathbb{C}^{n \times r}$, may be considered as the sum of the powers out of the rank-1 beamformers $\mathbf{w}_{i}$ :

$$
P(\theta)=\sum_{i=1}^{r} \mathbf{w}_{i}^{H} \mathbf{R} \mathbf{w}_{i}=\operatorname{tr}\left\{\mathbf{W}^{H} \mathbf{R W}\right\}
$$

This is consistent with the idea of multi-window spectrum estimators of [7] and [8].

\section{Multi-RAnK CAPON BEAMFORMING}

The standard Capon beamforming problem is posed as

$$
\min _{\mathbf{w}} P=\mathbf{w}^{H} \mathbf{R w} \quad \text { u.c. } \quad \mathbf{w}^{H} \mathbf{R}_{s s} \mathbf{w}=1
$$

where $\mathbf{R}_{s s}=\sigma_{s}^{2} \boldsymbol{\psi} \boldsymbol{\psi}^{H}$ is the rank-1 signal covariance matrix for the perfect planewave $\mathbf{s}=\boldsymbol{\psi} s$ with $E\left[s^{2}\right]=1$. Correspondingly, the Capon bearing spectrum and the Capon beamformer are

$$
\begin{aligned}
P_{C} & =\frac{\boldsymbol{\psi}^{H} \boldsymbol{\psi}}{\boldsymbol{\psi}^{H} \mathbf{R}^{-1} \boldsymbol{\psi}} \\
\mathbf{w} & =\frac{\mathbf{R}^{-1} \boldsymbol{\psi}}{\boldsymbol{\psi}^{H} \mathbf{R}^{-1} \boldsymbol{\psi}}
\end{aligned}
$$

A natural rank $-r$ generalization of (9) may seem to be

$$
\min _{\mathbf{W}} P=\operatorname{tr}\left\{\mathbf{W}^{H} \mathbf{R W}\right\} \quad \text { u.c. } \operatorname{tr}\left\{\mathbf{W}^{H} \mathbf{R}_{s s} \mathbf{W}\right\}=1
$$

where $\mathbf{W}=\left[\mathbf{w}_{1}, \ldots, \mathbf{w}_{r}\right] \in \mathbb{C}^{n \times r}$. However, under the trace constraint in (12) the columns of $\mathbf{W}$ do not have to be linearly independent. The problem will therefore decouple, and at the solution, the columns of $\mathbf{W}$ will be within scalar factors of the standard rank-1 Capon beamformer. Consequently, the resulting beamformer will still be rank-1 and no subspace expansion is obtained.

In order to force the columns of $\mathbf{W}$ to be linearly independent, we consider the constrained minimization problem

$$
\min _{\mathbf{W}} P=\operatorname{tr}\left\{\mathbf{W}^{H} \mathbf{R W}\right\} \text { u.c. } \mathbf{W}^{H} \mathbf{R}_{s s} \mathbf{W}=\mathbf{D}
$$

where $\mathbf{D} \in \mathbb{C}^{r \times r}$, with $r \leq p$, is a positive definite constraint matrix. To provide unit energy in the look direction, in analogy to the rank-1 Capon beamformer, we also require $\operatorname{tr}\{\mathbf{D}\}=$ 1. Note that $\mathbf{R}_{s s}$ and $\mathbf{D}$ are design parameters. The signal covariance matrix $\mathbf{R}_{s s}$ may be modelled as in (5) or (7). Later on we will present a few strategies for designing $\mathbf{D}$.

Consider an SVD of the presumed signal covariance matrix $\mathbf{R}_{s s}$ of the form

$$
\begin{aligned}
\mathbf{R}_{s s} & =\mathbf{U} \boldsymbol{\Sigma} \mathbf{U}^{H} \\
& =\left[\begin{array}{ll}
\mathbf{U}_{p} & \mathbf{U}_{\star}
\end{array}\right]\left[\begin{array}{cc}
\boldsymbol{\Sigma}_{p} & \mathbf{0} \\
\mathbf{0} & \mathbf{0}
\end{array}\right]\left[\begin{array}{c}
\mathbf{U}_{p}^{H} \\
\mathbf{U}_{\star}^{H}
\end{array}\right]=\mathbf{U}_{p} \boldsymbol{\Sigma}_{p} \mathbf{U}_{p}^{H}
\end{aligned}
$$

where $\mathbf{U}=\left[\begin{array}{ll}\mathbf{U}_{p} & \mathbf{U}_{\star}\end{array}\right] \in \mathbb{C}^{n \times n}$ is an orthogonal matrix, and $\boldsymbol{\Sigma}_{p}=\operatorname{diag}\left(\sigma_{1}, \ldots, \sigma_{p}\right)$ carries the nonzero eigenvalues of $\mathbf{R}_{s s}$, with $\sigma_{1} \geq \cdots \geq \sigma_{p}>0$. The matrix $\mathbf{U}_{p} \in \mathbb{C}^{n \times p}$ is an orthonormal basis for the presumed signal subspace and $\mathbf{U}_{\star} \in \mathbb{C}^{n \times(n-p)}$ is an orthonormal basis for the subspace orthogonal to $<\mathbf{U}_{p}>$. Further, consider an SVD of $\mathbf{D}$ of the form

$$
\mathbf{D}=\mathbf{A} \boldsymbol{\Delta} \mathbf{A}^{H}
$$

where $\mathbf{A}^{H} \mathbf{A}=\mathbf{A} \mathbf{A}^{H}=\mathbf{I}_{r}$ and $\boldsymbol{\Delta}=\operatorname{diag}\left(\delta_{1}, \ldots, \delta_{r}\right)$, with $\delta_{1} \geq \cdots \geq \delta_{r}>0$.

Solving the constrained quadratic minimization problem in (13) results in the multi-rank Capon bearing spectrum

$$
P_{M R C}(\theta)=\sum_{i=1}^{r} \frac{\delta_{i}(\theta)}{\lambda_{i}(\theta)}
$$


where $\lambda_{i}(\theta)$ is the $i$ th eigenvalue of $\mathbf{R}_{s s}^{H / 2} \mathbf{R}^{-1} \mathbf{R}_{s s}^{1 / 2}$, i.e.

$$
\lambda_{i}=\mathrm{ev}_{i}\left(\mathbf{R}_{s s}^{H / 2} \mathbf{R}^{-1} \mathbf{R}_{s s}^{1 / 2}\right)
$$

with $\lambda_{1} \geq \cdots \geq \lambda_{p}>0$. Here $\mathbf{R}_{s s}^{1 / 2} \in \mathbb{C}^{n \times p}$ is a squareroot of $\mathbf{R}_{s s}=\mathbf{R}_{s s}^{1 / 2} \mathbf{R}_{s s}^{H / 2}$ of the form $\mathbf{R}_{s s}^{1 / 2}=\mathbf{U}_{p} \boldsymbol{\Sigma}_{p}^{1 / 2} \mathbf{S}$, with $\boldsymbol{\Sigma}_{p}^{1 / 2}=\operatorname{diag}\left(\sqrt{\sigma_{1}}, \ldots, \sqrt{\sigma_{p}}\right)$ and $\mathbf{S} \in \mathbb{C}^{p \times p}$ an arbitrary orthogonal matrix. For detailed derivations of (16), and also the solution for the rank $-r$ Capon beamformer $\mathbf{W}$, see the Appendix.

Equation (16) shows that the spectrum $P_{M R C}$ is an harmonic average of the weighted eigenvalues of $\mathbf{R}_{s s}^{H / 2} \mathbf{R}^{-1} \mathbf{R}_{s s}^{1 / 2}$, where the weights are determined by the eigenvalues of the constraint matrix $\mathbf{D}$. In a signal-plus-interference-plus-noise model, $\mathbf{R}_{s s}^{H / 2} \mathbf{R}^{-1} \mathbf{R}_{s s}^{1 / 2}$ may be interpreted as the signal-tosignal-plus-interference-plus-noise matrix, and its eigenvalues $\lambda_{i}$ may be viewed as the per mode signal to total power ratios. Thus, the spectrum $P_{M R C}$ may be interpreted as an harmonic average of the weighted per mode signal to total power ratios, where the weights are designed to meet a set of quadratic constraints.

Remark: When $r=1, \mathbf{D}=1$, and $\mathbf{R}_{s s}=\boldsymbol{\psi} \boldsymbol{\psi}^{H}$, then the Capon spectrum in (16) becomes the standard Capon beamforming in (10).

\section{Designing the Constraint Matrix D}

The expression in (16) shows that the spectrum $P_{M R C}$ depends only on the eigenvalues of the constraint matrix D and not its eigenvectors. Thus, without loss of generality, we assume that $\mathbf{D}$ is diagonal: $\mathbf{D}=\operatorname{diag}\left(\delta_{1}, \ldots, \delta_{r}\right)$, with $\delta_{1} \geq \cdots \geq \delta_{r}>0$. In what follows three strategies for designing $\mathbf{D}$ are presented.

Choice 1: For the first choice, we consider

$$
\delta_{i}=\frac{1}{r}
$$

where the normalization by $r$ guarantees $\operatorname{tr}\{\mathbf{D}\}=1$. For this choice of $\mathbf{D}$, the spectrum $P$ reduces to the harmonic mean of the $\lambda_{i}$ :

$$
P_{M R C}=\sum_{i=1}^{r} \frac{1}{\lambda_{i}}
$$

The problem with this choice of $\mathbf{D}$ is that the spectrum $P_{M R C}$ is dominated by the smallest eigenvalue of $\mathbf{R}_{s s}^{H / 2} \mathbf{R}^{-1} \mathbf{R}_{s s}^{1 / 2}$. In other words, the mode with the smallest per mode signal to total power ratio has the most impact on the spectrum. Because of this, we anticipate that the performance for this choice of $\mathbf{D}$ degrades as rank increases. For $r=1$, the spectrum $P_{M R C}$ coincides with the spectrum of the standard Capon beamformer, i.e. $1 / \lambda_{1}$.

Choice 2: Another choice is to select the $\delta_{i}$ based upon the eigenvalues of the signal covariance matrix $\mathbf{R}_{s s}$. That is, to choose

$$
\delta_{i}=\frac{\sigma_{i}}{\sum_{i=1}^{r} \sigma_{i}}
$$

Unlike the first choice, in this case $\mathbf{D}$ is angle dependent, as the $\sigma_{i}=\operatorname{ev}_{i}\left(\mathbf{R}_{s s}\right)$ are functions of the look direction $\theta$. For this choice of $\mathbf{D}$, the spectrum becomes

$$
P_{M R C}=\frac{\sum_{i=1}^{r} \sigma_{i} / \lambda_{i}}{\sum_{i=1}^{r} \sigma_{i}}
$$

Again, for $r=1$ the spectrum reduces to that of the standard Capon beamformer, i.e. $1 / \lambda_{1}$.

Choice 3: Another way to design $\mathbf{D}$ is to think of the $\delta_{i}$ as weights that determine the contribution of the $\mathbf{w}_{i}$ in building the spectrum. One way to weigh the $\mathbf{w}_{i}$ is to choose the $\delta_{i}$ proportional to the eigenvalues of $\mathbf{R}_{s s}^{H / 2} \mathbf{R}^{-1} \mathbf{R}_{s s}^{1 / 2}$, i.e.

$$
\delta_{i}=\frac{\lambda_{i}}{\sum_{i=1}^{r} \lambda_{i}}
$$

This design for $\mathbf{D}$ is also angle dependent, as the $\lambda_{i}=$ $\mathrm{ev}_{i}\left(\mathbf{R}_{s s}^{H / 2}(\theta) \mathbf{R}^{-1} \mathbf{R}_{s s}^{1 / 2}(\theta)\right)$ are functions of the look direction $\theta$. This choice of $\mathbf{D}$ yields the spectrum

$$
P_{M R C}=\frac{1}{\sum_{i=1}^{r} \lambda_{i}}
$$

Unlike Choice 1, here the spectrum is not dominated by the smallest eigenvalue of $\mathbf{R}_{s s}^{H / 2} \mathbf{R}^{-1} \mathbf{R}_{s s}^{1 / 2}$, as each component in the summation in (23) contributes to the overall spectrum. Similar to the previous choices, for $r=1$ the spectrum reduces to that of the standard Capon beamformer, i.e. $1 / \lambda_{1}$.

\section{Simulation Results}

In this section, we present two numerical examples to evaluate the performance of the multi-rank Capon beamformers of Section III, and study how the design of the constraint matrix D affects the performance. We consider a uniform linear array with $n=20$ elements, and half-wavelength inter-element spacings. In the first example, three uncorrelated sources are incident on the array: two perfect planewaves (within the Rayleigh limit from each other) at angles $\theta_{1}=-\pi / 4$ and $\theta_{2}=$ $-\pi / 4.45$ and a wrinkled wavefront with angular bandwidth of $2 \pi \beta=.2 \pi$ (i.e. $\beta=0.1$ ), centered at $\theta_{3}=\pi / 8$. In the second example, the sources at $\theta_{1}=-\pi / 4$ and $\theta_{2}=-\pi / 4.45$ are wrinkled with $\beta=0.1$, and the one at $\theta_{3}=\pi / 8$ is a perfect planewave.

For the first example, the data covariance matrix $\mathbf{R}$ may be constructed as

$$
\mathbf{R}=\mathbf{R}_{s s}\left(\theta_{1}\right)+\mathbf{R}_{s s}\left(\theta_{2}\right)+\mathbf{R}_{s s}\left(\theta_{3}\right)+\mathbf{R}_{n n}
$$

where $\mathbf{R}_{s s}\left(\theta_{i}\right)=\boldsymbol{\psi}\left(\theta_{i}\right) \sigma_{s, i}^{2} \boldsymbol{\psi}\left(\theta_{i}\right)^{H}, i=1,2$ are covariance matrices for the two planewaves, and $\mathbf{R}_{s s}\left(\theta_{3}\right)$ is the covariance matrix of the wrinkled wavefront:

$$
\mathbf{R}_{s s}\left(\theta_{3}\right)=\mathbf{D}\left(\theta_{3}\right)\left[\int_{-\pi \beta}^{\pi \beta} \boldsymbol{\psi}\left(\theta^{\prime}\right) \boldsymbol{\psi}\left(\theta^{\prime}\right)^{H} S\left(\theta^{\prime}\right) \frac{d \theta^{\prime}}{2 \pi \beta}\right] \mathbf{D}\left(\theta_{3}\right)^{H}
$$


The matrix $\mathbf{R}_{n n}=\sigma_{n}^{2} \mathbf{I}$ is the noise covariance. We choose $\sigma_{s, i}^{2}=1(i=1,2)$, and $\sigma_{n}^{2}=0.01$, for input SNR of $7 \mathrm{~dB}$ at each sensor. In the second example, we just reverse the roles of $\mathbf{R}_{s s}\left(\theta_{1}\right), \mathbf{R}_{s s}\left(\theta_{2}\right)$, and $\mathbf{R}_{s s}\left(\theta_{3}\right)$. This is the way the data covariance matrix $\mathbf{R}$ is constructed. For beamforming, the signal covariance matrix $\mathbf{R}_{s s}(\theta)$ is an angle dependent design parameter, which is computed using (5), at each look direction.

Example 1: Figure 1(a) shows the bearing spectra for the standard Bartlett and Capon beamformers. The locations of the sources are illustrated by vertical solid lines. The two sources at $\theta_{1}=-\pi / 4$ and $\theta_{2}=-\pi / 4.45$ are the perfect planewaves, and the one at $\theta_{3}=\pi / 8$ is the wrinkled wavefront. Figures 1(b)-(d) show the bearing spectra for the multi-rank Capon beamformers for the three choices of $\mathbf{D}$, discussed in Section IV. In each figure, the bearing spectra are plotted for rank-1 to rank-3 Capon beamformers. As can be seen the standard Bartlett and Capon beamformers offer low detectability for the wrinkled wavefront. In addition, it seems that the standard Capon beamformer tends to treat the wrinkled wavefront at $\theta_{3}=\pi / 8$ as if there were multiple planewaves at nearby angles, instead of a single non-planar wave. The multi-rank Capon beamformers, on the other hand, offer high detectability for the wrinkled wavefront, but show poor performance for the perfect planewaves. It is clearly seen that as the rank of the Capon beamformer increases the bearing spectra around the source locations become wider, and hence resolution is lost. In addition, the broadening of the bearing spectra with the increase in rank is most significant for the first choice of $\mathbf{D}$ (i.e. $\mathbf{D}=\mathbf{I}$ ) and least significant for the third choice of $\mathbf{D}$ (i.e. the one in (22)). Thus, it seems that the third choice for $\mathrm{D}$ results in the smallest loss of resolution compared to the other two.

Example 2: In this example, the perfect planewaves at $\theta_{1}=-\pi / 4$ and $\theta_{2}=-\pi / 4.45$ are replaced by two wrinkled wavefront with $\beta=0.1$, and the wrinkled wavefront at $\theta_{3}=$ $\pi / 8$ is replaced by a perfect planewave. Figure 2(a) shows the corresponding bearing spectra for the standard Bartlett and Capon beamformers. The bearing spectra for the multirank Capon beamformers are plotted in Figures 2(b)-(d). As can be seen, the standard Capon beamformer cannot resolve the two winkled wavefronts, due to their angular spread. The multi-rank Capon beamformers, however, are able to resolve them. Similar to the previous example, the multi-rank Capon beamformers offer higher detectability for the wrinkled wavefronts, but lower detectability for the perfect planewaves, compared to the standard Capon and Bartlett beamformers. The effects of rank and choice of $\mathbf{D}$ on the performance of the multi-rank Capon beamformers are also similar to those in Example 1. That is, the increase in rank results in the loss of resolution. Further, the third choice for D (Fig. 2(d)) seems to offer the narrowest bearing response, while maintaining high detectability for the wrinkled wavefronts.

\section{CONCLUSIONS}

A multi-rank generalization of the Capon beamformer has been presented. The idea is to expand the beamforming subspace to accommodate model mismatches. The generalization involves solving a quadratically-constrained quadratic minimization problem and designing a positive definite constraint matrix. Three strategies for designing the constraint matrix have been discussed. Two simulation examples have been presented, suggesting that multi-rank Capon beamformers provide robustness to model mismatches at the expense of resolution. However, these results are preliminary. It must be emphasized that, so far, our other simulation results have been mixed, making it difficult to make a definitive claim about the scenarios for which the multi-rank Capon beamformers are preferred. Our experiments with constraints continue, as do our comparisons with other robust beamformers.

\section{APPENDIX}

Since $\mathbf{U}=\left[\begin{array}{ll}\mathbf{U}_{p} & \mathbf{U}_{\star}\end{array}\right]$ is a basis for $\mathbb{C}^{n}$, without loss of generality, we express $\mathbf{W} \in \mathbb{C}^{n \times r}$ as

$$
\mathbf{W}=\mathbf{U B}=\left[\begin{array}{ll}
\mathbf{U}_{p} & \mathbf{U}_{\star}
\end{array}\right]\left[\begin{array}{l}
\mathbf{B}_{p} \\
\mathbf{B}_{\star}
\end{array}\right]=\mathbf{W}_{p}+\mathbf{W}_{\star}
$$

where $\mathbf{B}_{p} \in \mathbb{C}^{p \times r}$ and $\mathbf{B}_{\star} \in \mathbb{C}^{(n-p) \times r}$ are matrices that build the orthogonal parts of $\mathbf{W}$, i.e. $\mathbf{W}_{p}=\mathbf{U}_{p} \mathbf{B}_{p}$ and $\mathbf{W}_{\star}=$ $\mathbf{U}_{\star} \mathbf{B}_{\star}$, inside $<\mathbf{U}_{p}>$ and $\left\langle\mathbf{U}_{\star}>\right.$.

We start by satisfying the quadratic constraint $\mathbf{W}^{H} \mathbf{R}_{s s} \mathbf{W}=\mathbf{D}$. Using the SVD in (14) and replacing for $\mathbf{W}$ from (26), we may simplify the constraint to

$$
\left[\begin{array}{ll}
\mathbf{B}_{p}^{H} & \mathbf{B}_{\star}^{H}
\end{array}\right]\left[\begin{array}{cc}
\boldsymbol{\Sigma}_{\boldsymbol{p}} & \mathbf{0} \\
\mathbf{0} & \mathbf{0}
\end{array}\right]\left[\begin{array}{l}
\mathbf{B}_{p} \\
\mathbf{B}_{\star}
\end{array}\right]=\mathbf{D}
$$

or equivalently

$$
\mathbf{B}_{p}^{H} \boldsymbol{\Sigma}_{p} \mathbf{B}_{p}=\mathbf{D} \text {. }
$$

Therefore, $\mathbf{B}_{p}$ must be of the form

$$
\mathbf{B}_{p}=\boldsymbol{\Sigma}_{p}^{-H / 2} \mathbf{E D}^{H / 2}
$$

where $\boldsymbol{\Sigma}_{p}^{-H / 2}=\boldsymbol{\Sigma}_{p}^{-1 / 2}=\operatorname{diag}\left(1 / \sqrt{\sigma_{1}}, \ldots, 1 / \sqrt{\sigma_{r}}\right)$ is a square-root of $\boldsymbol{\Sigma}_{p}^{-1}=\boldsymbol{\Sigma}_{p}^{-H / 2} \boldsymbol{\Sigma}_{p}^{-1 / 2}$, and $\mathbf{E} \in \mathbb{C}^{p \times r}$ is a slice of an orthogonal matrix, yet to be determined. Thus, $\mathbf{E}^{H} \mathbf{E}=\mathbf{I}_{p}$ and $\mathbf{E E}^{H}=\mathbf{P}_{\mathbf{E}}$, where $\mathbf{P}_{\mathbf{E}}$ is an orthogonal projection onto $\left\langle\mathbf{E}>\right.$. The matrix $\mathbf{D}^{1 / 2} \in \mathbb{C}^{r \times r}$ is a squareroot of $\mathbf{D}=\mathbf{D}^{1 / 2} \mathbf{D}^{H / 2}$. From the SVD in (15), one possible choice for $\mathbf{D}^{1 / 2}$ is

$$
\mathbf{D}^{1 / 2}=\mathbf{A} \boldsymbol{\Delta}^{1 / 2}
$$

where $\boldsymbol{\Delta}^{1 / 2}=\operatorname{diag}\left(\sqrt{\delta_{1}}, \ldots, \sqrt{\delta_{r}}\right)$. We could post-multiply any $r \times r$ orthogonal matrix on the right hand side of (30). However, the product of such a matrix with $\mathbf{E}$ in (29) would still be a slice of an orthogonal matrix. Thus, without loss of generality, we assume that such a matrix is absorbed in $\mathbf{E}$.

Using (30), we may write $\mathbf{B}_{p}$ as

$$
\mathbf{B}_{p}=\boldsymbol{\Sigma}_{p}^{-H / 2} \mathbf{E D}^{H / 2}=\boldsymbol{\Sigma}_{p}^{-H / 2} \mathbf{E} \boldsymbol{\Delta}^{H / 2} \mathbf{A}^{H} .
$$




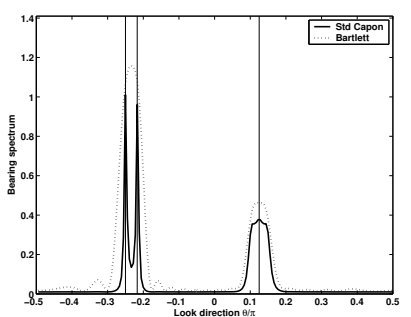

(a)

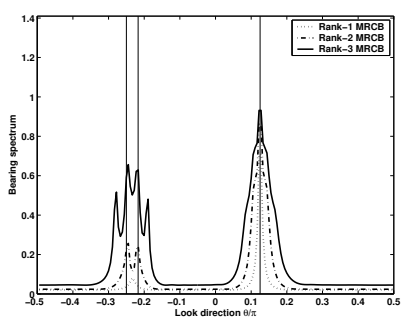

(b)

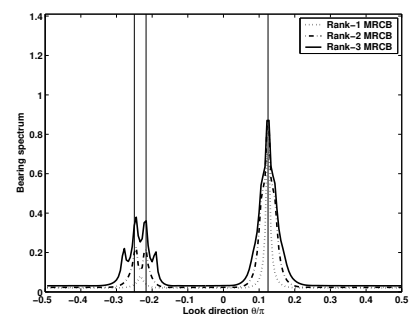

(c)

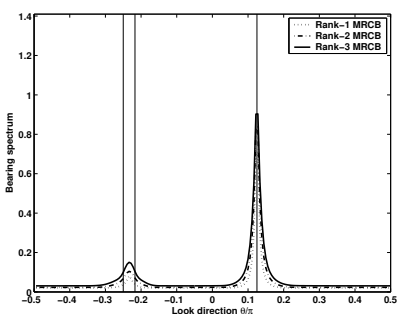

(d)

Fig. 1. Bearing spectra for the standard Bartlett and Capon beamformers, and multi-rank Capon beamformers. Going from (b) to (c) to (d) the multi-rank Capon beamformers are constructed with the constraint matrix $\mathbf{D}$ being chosen as in (18), (20), and (22), respectively. The sources are two perfect planewaves (within the Rayleigh limit from each other) at $\theta_{1}=-\pi / 4$ and $\theta_{2}=-\pi / 4.45$, and a wrinkled wavefront, with $\beta=0.1$, centered at $\theta_{3}=\pi / 8$.

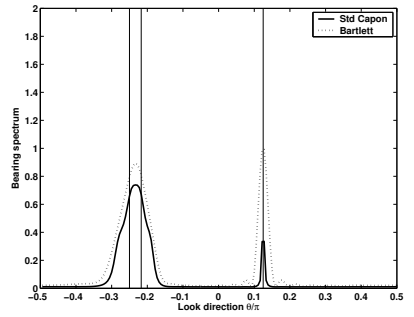

(a)

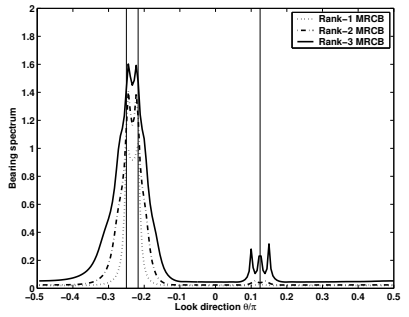

(b)

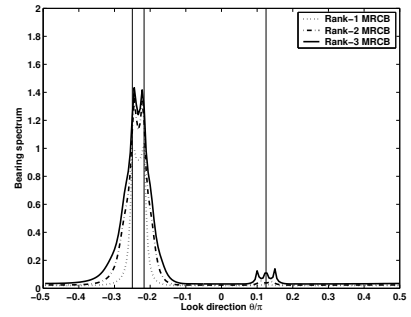

(c)

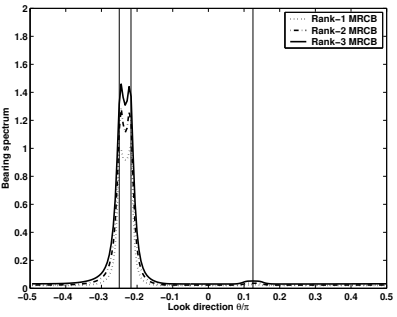

(d)

Fig. 2. Bearing spectra for the standard Bartlett and Capon beamformers, and multi-rank Capon beamformers. Going from (b) to (c) to (d) the multi-rank Capon beamformers are constructed with the constraint matrix $\mathbf{D}$ being chosen as in (18), (20), and (22), respectively. The sources are two wrinkled wavefronts, with $\beta=0.1$, centered at $\theta_{1}=-\pi / 4$ and $\theta_{2}=-\pi / 4.45$, and a perfect planewave at $\theta_{3}=\pi / 8$.

Inserting $\mathbf{W}=\mathbf{U}_{p} \mathbf{B}_{p}+\mathbf{U}_{\star} \mathbf{B}_{\star}$ in $P=\operatorname{tr}\left\{\mathbf{W}^{H} \mathbf{R W}\right\}$ yields

$$
\begin{aligned}
P=\operatorname{tr} & \left(\mathbf{R}^{H / 2} \mathbf{U}_{p} \mathbf{B}_{p}+\mathbf{R}^{H / 2} \mathbf{U}_{\star} \mathbf{B}_{\star}\right)^{H} \\
& \left.\left(\mathbf{R}^{H / 2} \mathbf{U}_{p} \mathbf{B}_{p}+\mathbf{R}^{H / 2} \mathbf{U}_{\star} \mathbf{B}_{\star}\right)\right\} .
\end{aligned}
$$

Assuming $\mathbf{B}_{p}$ is fixed, we minimize $P$ with respect to $\mathbf{B}_{\star}$. This results in a least squares solution for $\mathbf{B}_{\star}$ of the form

$$
\mathbf{B}_{\star}=-\left(\mathbf{R}^{H / 2} \mathbf{U}_{\star}\right)^{\dagger} \mathbf{R}^{H / 2} \mathbf{U}_{p} \mathbf{B}_{p}
$$

where $(\cdot)^{\dagger}$ denotes the Moore-Penrose pseudo inverse [13].

Plugging $\mathbf{B}_{\star}$ in (32) yields

$$
\begin{aligned}
P & =\operatorname{tr}\left\{\mathbf{B}_{p}^{H} \mathbf{U}_{p}^{H} \mathbf{R}^{1 / 2}\left[\mathbf{I}-\mathbf{P}_{\mathbf{R}^{H / 2} \mathbf{U}_{\star}}\right] \mathbf{R}^{H / 2} \mathbf{U}_{p} \mathbf{B}_{p}\right\} \\
& =\operatorname{tr}\left\{\mathbf{B}_{p}^{H} \mathbf{U}_{p}^{H} \mathbf{R}^{1 / 2} \mathbf{P}_{\mathbf{R}^{H / 2} \mathbf{U}_{\star}}^{\perp} \mathbf{R}^{H / 2} \mathbf{U}_{p} \mathbf{B}_{p}\right\}
\end{aligned}
$$

where $\mathbf{P}_{\mathbf{R}^{H / 2} \mathbf{U}_{\star}}=\mathbf{R}^{H / 2} \mathbf{U}_{\star}\left(\mathbf{R}^{H / 2} \mathbf{U}_{\star}\right)^{\dagger}$ is an orthogonal projection onto $<\mathbf{R}^{H / 2} \mathbf{U}_{\star}>$, and $\mathbf{P}_{\mathbf{R}^{H / 2} \mathbf{U}_{\star}}^{\perp}=\mathbf{I}-\mathbf{P}_{\mathbf{R}^{H / 2} \mathbf{U}_{\star}}$ is an orthogonal projection onto the subspace orthogonal to $<\mathbf{R}^{H / 2} \mathbf{U}_{\star}>$. Let

$$
\mathbf{P}_{\mathbf{R}^{-1 / 2} \mathbf{U}_{p}}=\mathbf{R}^{-1 / 2} \mathbf{U}_{p}\left(\mathbf{U}_{p}^{H} \mathbf{R}^{-1} \mathbf{U}_{p}\right)^{-1} \mathbf{U}_{p}^{H} \mathbf{R}^{-H / 2}
$$

be the orthogonal projection onto $<\mathbf{R}^{-1 / 2} \mathbf{U}_{p}>$. Then, it is easy to show that

$$
\mathbf{P}_{\mathbf{R}^{H / 2} \mathbf{U}_{\star}}^{\perp}=\mathbf{P}_{\mathbf{R}^{-1 / 2} \mathbf{U}_{p}}
$$

as $\mathbf{P}_{\mathbf{R}^{-1 / 2} \mathbf{U}_{p}} \mathbf{P}_{\mathbf{R}^{H / 2} \mathbf{U}_{\star}}=\mathbf{0}$, and $\mathbf{P}_{\mathbf{R}^{-1 / 2} \mathbf{U}_{p}}$ and $\mathbf{P}_{\mathbf{R}^{H / 2} \mathbf{U}_{\star}}$ together span the entire $\mathbb{C}^{n}$.

Using (35) and (36), we may write (34) as

$$
P=\operatorname{tr}\left\{\mathbf{B}_{p}^{H}\left(\mathbf{U}_{p}^{H} \mathbf{R}^{-1} \mathbf{U}_{p}\right)^{-1} \mathbf{B}_{p}\right\}
$$

Plugging $\mathbf{B}_{p}=\boldsymbol{\Sigma}_{p}^{-H / 2} \mathbf{E D}^{H / 2}$ in (37) yields

$$
P=\operatorname{tr}\left\{\mathbf{E}^{H}\left(\boldsymbol{\Sigma}_{p}^{H / 2} \mathbf{U}_{p}^{H} \mathbf{R}^{-1} \mathbf{U}_{p} \boldsymbol{\Sigma}_{p}^{1 / 2}\right)^{-1} \mathbf{E} \mathbf{D}^{H / 2} \mathbf{D}^{1 / 2}\right\}
$$

Replacing $\mathbf{D}^{1 / 2}$ from (30) results in

$$
P=\operatorname{tr}\left\{\mathbf{E}^{H}\left(\boldsymbol{\Sigma}_{p}^{H / 2} \mathbf{U}_{p}^{H} \mathbf{R}^{-1} \mathbf{U}_{p} \boldsymbol{\Sigma}_{p}^{1 / 2}\right)^{-1} \mathbf{E} \boldsymbol{\Delta}\right\}
$$

We now minimize $P$ with respect $\mathbf{E}$, keeping in mind that $\mathbf{E}^{H} \mathbf{E}=\mathbf{I}_{r}$. Define $\mathbf{M}$ as

$$
\mathbf{M}=\boldsymbol{\Sigma}_{p}^{H / 2} \mathbf{U}_{p}^{H} \mathbf{R}^{-1} \mathbf{U}_{p} \boldsymbol{\Sigma}_{p}^{1 / 2}
$$

and let $\mu_{i}$ be the $i$ th eigenvalue of $\mathbf{E}^{H} \mathbf{M}^{-1} \mathbf{E}$ :

$$
\mu_{i}=\mathrm{ev}_{i}\left(\mathbf{E}^{H} \mathbf{M}^{-1} \mathbf{E}\right)
$$

where $0<\mu_{1} \leq \cdots \leq \mu_{r}$. Inserting $\mathbf{M}$ in (39) and using a majorization result for matrix trace $[14]^{1}$ yields the inequality

$$
P=\operatorname{tr}\left\{\mathbf{E}^{H} \mathbf{M}^{-1} \mathbf{E} \boldsymbol{\Delta}\right\} \geq \sum_{i=1}^{r} \mu_{i} \delta_{i} .
$$

Let

$$
\mathbf{M}=\mathbf{V} \boldsymbol{\Lambda} \mathbf{V}^{H}
$$

be a SVD of $\mathbf{M}$, where $\boldsymbol{\Lambda}=\operatorname{diag}\left(\lambda_{1}, \ldots, \lambda_{p}\right), \lambda_{1} \geq \cdots \geq$ $\lambda_{p}>0$ carries the eigenvalues of $\mathbf{M}$, and the matrix $\mathbf{V}$ is orthogonal: $\mathbf{V}^{H} \mathbf{V}=\mathbf{V} \mathbf{V}^{H}=\mathbf{I}_{p}$. Then,

$$
\begin{aligned}
& \mathbf{M}^{-1}=\mathbf{V} \boldsymbol{\Lambda}^{-1} \mathbf{V}^{H} ; \\
& \mathbf{\Lambda}^{-1}=\operatorname{diag}\left(\frac{1}{\lambda_{1}}, \ldots, \frac{1}{\lambda_{p}}\right)
\end{aligned}
$$

${ }^{1}$ Majorization result for matrix trace: Let $\mathbf{T}$ and $\mathbf{S}$ be $r \times r$ nonnegative definite matrices, with eigenvalues $0 \leq t_{1} \leq \cdots \leq t_{r}$ and $s_{1} \geq \cdots \geq s_{r} \geq$ 0 , respectively. Then, $\operatorname{tr}\{\mathbf{T S}\} \geq \sum_{i=1}^{r} t_{i} s_{i}$. 
is an eigenvalue decomposition of $\mathbf{M}^{-1}$, in which the eigenvalues are arranged in ascending order: $0<1 / \lambda_{1} \leq \cdots \leq 1 / \lambda_{p}$.

Since $\mathbf{E}$ is a slice of an orthogonal matrix, from Poincare's separation theorem $[15]^{2}$, the eigenvalues of $\mathbf{M}^{-1}$ and $\mathbf{E}^{H} \mathbf{M}^{-1} \mathbf{E}$ satisfy the inequality

$$
\frac{1}{\lambda_{i}} \leq \mu_{i} \leq \frac{1}{\lambda_{p-r+i}}, \quad i=1, \ldots, r .
$$

The left hand side equality in (45) holds when $\mathbf{E}$ diagonalizes $\mathbf{M}$, i.e. when $\mathbf{E}$ is a slice of $\mathbf{V}$.

Combining (42) and (45), and plugging in $\mathbf{M}=\mathbf{V} \boldsymbol{\Lambda} \mathbf{V}^{H}$ yields

$$
P=\operatorname{tr}\left\{\mathbf{E}^{H} \mathbf{V} \boldsymbol{\Lambda}^{-1} \mathbf{V}^{H} \mathbf{E} \boldsymbol{\Delta}\right\} \geq \sum_{i=1}^{r} \frac{\delta_{i}}{\lambda_{i}}
$$

The equality holds when $\mathbf{E}^{H} \mathbf{V}=\left[\begin{array}{ll}\mathbf{I}_{r} & \mathbf{0}\end{array}\right]$ or equivalently

$$
\mathbf{E}=\mathbf{V}\left[\begin{array}{c}
\mathbf{I}_{r} \\
\mathbf{0}
\end{array}\right]=\mathbf{V}_{r}
$$

where $\mathbf{V}_{r} \in \mathbb{C}^{p \times r}$ carries the first $r$ columns of $\mathbf{V}$. Finally, the minimum value of $P$ is $P_{\min }=P_{M R C}$,

$$
P_{M R C}=\sum_{i=1}^{r} \frac{\delta_{i}}{\lambda_{i}}
$$

Let $\mathbf{S}$ be an arbitrary orthogonal matrix. Then the eigenvalues of $\mathbf{S M} \mathbf{S}^{H}=\mathbf{S} \boldsymbol{\Sigma}_{p}^{H / 2} \mathbf{U}_{p}^{H} \mathbf{R}^{-1} \mathbf{U}_{p} \boldsymbol{\Sigma}_{p}^{1 / 2} \mathbf{S}^{H}$ are the same as the eigenvalues of $\mathbf{M}=\boldsymbol{\Sigma}_{p}^{H / 2} \mathbf{U}_{p}^{H} \mathbf{R}^{-1} \mathbf{U}_{p} \boldsymbol{\Sigma}_{p}^{1 / 2}$. Noting that $\mathbf{R}_{s s}^{1 / 2}=\mathbf{U}_{p} \boldsymbol{\Sigma}_{p}^{1 / 2} \mathbf{S}^{H}$ is a square-root of $\mathbf{R}_{s s}$, i.e. $\mathbf{R}_{s s}=$ $\mathbf{R}_{s s}^{1 / 2} \mathbf{R}_{s s}^{H / 2}$, we may express $\lambda_{i}$ as

$$
\lambda_{i}=\operatorname{ev}_{i}\left(\mathbf{R}_{s s}^{H / 2} \mathbf{R}^{-1} \mathbf{R}_{s s}^{1 / 2}\right) .
$$

This completes the derivation of (16).

To determine the solution to $\mathbf{W}=\mathbf{W}_{p}+\mathbf{W}_{\star}$, note that from (26), (31), and (47), $\mathbf{W}_{p}$ is obtained as

$$
\mathbf{W}_{p}=\mathbf{U}_{p} \mathbf{B}_{p}=\mathbf{U}_{p} \boldsymbol{\Sigma}_{p}^{-H / 2} \mathbf{V}_{r} \mathbf{D}^{H / 2} .
$$

Using (26) and (33), we may write $\mathbf{W}_{\star}$ as

$$
\mathbf{W}_{\star}=\mathbf{U}_{\star} \mathbf{B}_{\star}=-\mathbf{K} \mathbf{W}_{p}
$$

where

$$
\mathbf{K}=\mathbf{R}^{-H / 2} \mathbf{P}_{\mathbf{R}^{H / 2} \mathbf{U}_{\star}} \mathbf{R}^{H / 2}=\mathbf{R}^{-H / 2} \mathbf{P}_{\mathbf{R}^{-1 / 2} \mathbf{U}_{p}}^{\perp} \mathbf{R}^{H / 2}
$$

is an oblique projection [16],[17]. Therefore, $\mathbf{W}_{\star}$ is obtained by obliquely projecting $\mathbf{W}_{p}$ using $-\mathbf{K}$. Using (50) and (51), the rank $-r$ Capon beamformer $\mathbf{W}$ is

$$
\mathbf{W}=\mathbf{W}_{p}+\mathbf{W}_{\star}=(\mathbf{I}-\mathbf{K}) \mathbf{W}_{p} .
$$

${ }^{2}$ Poincare's Separation Theorem: Let $\mathbf{S} \in \mathbb{C}^{p \times p}$ be a Hermitian matrix with eigenvalues $0 \leq s_{1} \leq \cdots \leq s_{p}, \mathbf{X} \in \mathbb{C}^{p \times r}(r \leq p)$ a slice of an orthogonal matrix, i.e. $\mathbf{X}_{r}^{H} \overline{\mathbf{X}}_{r}=\overline{\mathbf{I}}_{r}$, and $0 \leq t_{1} \leq \cdots \leq t_{r}$ the eigenvalues of $\mathbf{X}^{H} \mathbf{S X}$. Then, $s_{i} \leq t_{i} \leq s_{p-r+i}, i=1, \ldots, r \leq p$.

\section{REFERENCES}

[1] H. Cox, "Resolving power and sensitivity to mismatch of optimum array processors," J. Acoust. Soc. Amer., vol. 54, pp. 771-758, 1973.

[2] D. D. Feldman and L. J. Griffiths, "A projection approach to robust adaptive beamforming," IEEE Trans. Signal Processing, vol. 42, pp. 867-876, Apr. 1994.

[3] H. Cox, R. M. Zeskind, and M. M. Owen, "Robust adaptive beamforming," IEEE Trans. Acoust. Speech Signal Process., vol. 2, pp. 1365-1376, Oct. 1987.

[4] S. Shahbazpanahi, A. B. Gershman, Z. Luo, and K. M. Wong, "Robust adaptive beamforming for general-rank signal models," IEEE Trans. Signal Processing, vol. 51, pp. 2257-2269, Sept. 2003.

[5] J. Li, P. Stoica, and Z. Wang, "On robust Capon beamforming and diagonal loading," IEEE Trans. on Signal Processing, vol. 51, pp. 1702 1715 , July 2003.

[6] R. G. Lorenz and S. P. Boyd, "Robust minimum variance beamforming," IEEE Trans. Signal Processing, vol. 53, pp. 1684-1696, May 2005.

[7] D. J. Thomson, "Spctrum estimation and harmonic analysis," Proc. IEEE, vol. 70, pp. 1055-1096, Sept. 1982.

[8] C. T. Mullis and L. L. Scharf, "Quadratic estimators of the power spectrum," in Chapter One of Advances in Spectrum Estimation, S. Haykin, Ed. Englewood Cliffs, NJ: Prentice Hall, 1990.

[9] H. Cox, "Sensitivity considerations in adaptive beamforming," Signal Processing (Proc. NATO Adv. Study Inst., Loughborough, U.K., Aug. 1972), pp. 619-645, 1973.

[10] _ , "Interrelated problems in estimation and detection I and II," in Proc. NATO Adv. Study Inst. Signal Processing with Emphasis on Underwater Acoust., Enschede, The Netherlands, Aug. 1973.

[11] M. Lundberg, L. L. Scharf, and A. Pezeshki, "Multi-rank Capon beamforming," in Conf. Rec. Thrity-Eighth Asilomar Conf. Signals, Syst., Comput., Pacific Grove, CA, Nov. 7-10 2004.

[12] A. Pezeshki, L. L. Scharf, and H. Cox, "On linearly and quadratically constrained quadratic minimization problems for beamforming and diversity combining," Linear Algebra and Its Applications, under preparation.

[13] G. H. Golub and C. F. Van Loan, Matrix Computations, 3rd ed. Baltimore, MD: John Hopkins Univ. Press, 1996.

[14] A. W. Marshall and I. Olkin, Inequalities: Theory of Majorization and Its Applications. New York: Academic Press, 1979.

[15] H. Lütkepohl, Handbook of Matrices. Wiley, 1996.

[16] S. Kayalar and H. L. Weinert, "Oblique projections: Formulas, algorithms and error bounds," Math. Contr. Signals Syst., vol. 2, pp. 33-45, 1989.

[17] R. T. Behrens and L. L. Scharf, "Signal processing applications of oblique projection operators," IEEE Trans. Signal Processing, vol. 42, pp. 1413-1424, June 1994. 